Volume No. 1, Issue No. 2 (April- June), pp. 1-9

\title{
Algorithm for Using Codes In Place Of Facial Images during Image Processing In Large Databases and Data Warehouses to Reduce Storage, Enhance Efficiency and Processing Speed
}

\author{
Aftab Ahmad Malik and Mujtaba Asad
}

\begin{abstract}
The main purpose of this work is to assign codes to the facial image stored in large databases / Data warehouses, the images require large amount of storage as compared to a numeric code. Moreover, when images are compared to images, the process is more time consuming as compared to the numeric codes. It is proposed to keep up all images in a separate database file along with their codes. The codes of facial images may be stored in master Database/ Data warehouse for all records. The search queries can be processed using the numeric codes. In this manner the Time Complexity and Space Complexity are reduced considerably. Whenever, any image is received for searching its record, first its code is obtained by proposed algorithm and then this code is used to search the record from database/Data Warehouse making entire procedure faster and efficient.
\end{abstract}

Keywords: - Image processing, coding, facial images, Numeric Codes, Database / Data Warehouse

\section{INTRODUCTION}

There exist several techniques to analyze and synthesize the human facial images. The facial images are analyzed using various poses, especially the front or side poses. The oblique left or oblique right facial images require more rigorous computing. It is usual practice to store the front pose of facial images in Databases/Data Warehouses keeping in view the posture and position of eyes pair, chin and nose in

Prof. Dr. Aftab Ahmad Malik, Ph.D. University of Kent, Canterbury, England

Department of Computer Science, Lahore Garrison University Lahore, Pakistan dr_aftab_malik@yahoo.com

Mujtaba Asad, Ph.D. Scholar, UET, Lahore, Pakistan

Lecturer (CS), Lahore Garrison University Lahore, Pakistan 
such a way that right and left side of
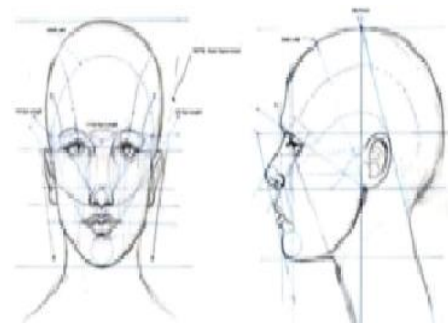

Figure 1: Face Features

the face is symmetrically visible and covered up in the image.

Proposed \& devised an efficient algorithm for Coding Person's Names in large Databases/ Data warehouses to enhance Processing speed, Efficiency and to reduce Storage Requirements. [1] Allocates numeric codes to Names (First name, Middle name, Last name) using three numeric characters for each instead of 40 Alpha-Characters, which are normally reserved for one complete name. A similar approach shall be employed for facial image coding in this paper. The philosophy of [1] is applied to images in this paper to reduce Time Complexity and Space Complexity. [2] Describes a method to assign codes i.e. Unique ID for face recognition using openCV from open source. It allocates code in the situation, for example, when person is waiting in front of camera for several exposures and the algorithm allocates codes to each pose.[3] Describes uses and procedures of OpenCV. It provides built in Library functions for real time image processing. The imgproc module provides basic and important image processing Techniques/Algorithms for filtering, image transformations and conversions.

Voila Jones Algorithm is discussed in [4], which identifies and detects various parts of the facial image, the eyes, nose and mouth using common characteristics of the face. [2] Claims that $99 \%$ accuracy in image processing can be achieved in frontpose face image. However, the accuracy is considerably reduced with glasses, oblique face position and changed hairstyle, increasing or decreasing light illumination. For unique images most important factors are depth of jaws, distance between eyes, nose width and length of cheekbones for changing image into digital code. Different patterns to code the finger prints have been presented in [5] in order to use these codes in the software for storage and retrieval of criminal information. 
Various issues regarding facial Definition: Elastic Bunch Graph patterns and physical appearance Matching (EBGM) [8]: This have been elaborated in [6]. For algorithm is used in computer vision facial image processing and coding, to recognize the objects of an image we are interested in the front pose on a graph representation derived face pattern only. The front pose from other images. The Elastic facial image from [7] also provides Bunch Graph Matching returns the the points for measurement of similarity value, the positions of the distances as shown below:

Moreover, the points of measurement of various distances are illustrated from the following figure [5]:
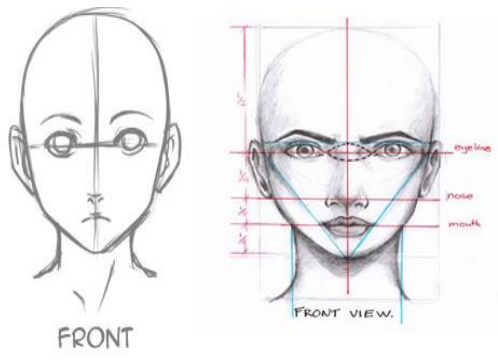

Figure 2: Points of Measurement of Various Distances

The following images [7] also clarify the points of measurements of distances:
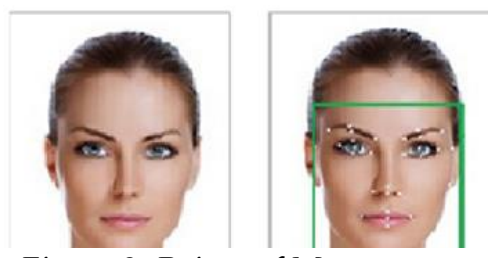

Figure 3: Points of Measurement (Depicted as Points nodes in image, which helps in creating an image graph.

Definition: Gabor Filters [9] are used in image processing with Gaussian envelop function for texture analysis. We can express profile of simple cell using a 2-D Gabor function.

\section{Definition: Biometrics:}

This technology is extensively and commonly used in forensic to analyze for measurement and examine the characteristics of humans like DNA, irises, and voices and face images. The related information can be converted into digital form, encrypted and stored in a database. The algorithm can translate the given image into a 
digital code.

It is remarkable that God has created humans with different physical appearances, unique DNA, finger prints and iris, which do not change. Moreover, all these characteristics

\section{METHODOLOGY}

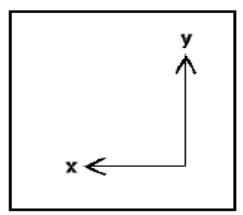

Manhattan

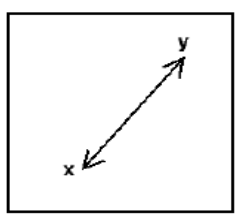

Euclidean

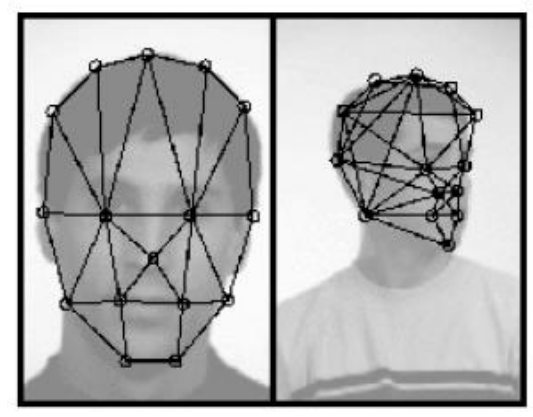

Figure 4 Grids for Face Finding

can be measured.

\section{Applications of Biometrics:}

There are several biometric systems designed to identify humans by matching the characteristics from the database or Data warehouse. The employee's roll call system is one such example, others in daily life are computer login, internet access, ATM forensic applications such as used in Criminal Information system elaborated and designed in [5][6]. The Face Recognition by Elastic Bunch Graph Matching has been discussed in [10].
Figure 5: Representation of Manhattan and Euclidean Distances

A grid of points is launched as

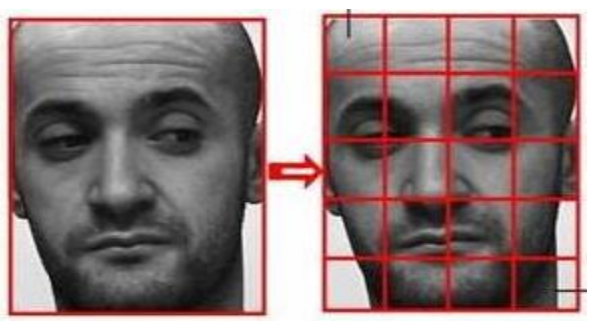

Figure 6: Division of Face Image into Blocks

shown, then a bunch graph is constructed for recognition, it works with Instructions on getting FERET database. The purpose of FERET is to design automatic Face Recognition System. For Enhanced Security of Digital Images [12][13] can be used developed by the second author of this paper,

\section{Assigning code to facial image:}

There are eighty nodal points (or end points) on human face by means of which the digital code called face 
print can be generated by biometric // calculate distance between Left eye software as shown above. The center (LEC) and nose tip (NT) distance between relevant points is computed for code generation. The assumptions for Algorithm such as given below:

- Front view of the image

- Image is captured from fixed distance from the camera

Double distanceBetweenLECNT = Math.Sqrt((facialFeatures [2].yfacialFeatures $[0] \cdot y)^{\wedge}$ 2) + ((facialFeatures[2].x facialFeatures [0].x) ^ 2));

In fact, the pixels values of points on the face, (xi, yi) are used to generate a unique 14 digits code, which is to be used as a key in database instead of image to speed up the process.

$$
\begin{aligned}
& d_{E}(x, y) \\
& =\sqrt{\left(x_{1}-y_{1}\right)^{2}+\left(x_{2}-y_{2}\right)^{2}} \\
& =\sqrt{\sum_{i=1}^{n}\left(x_{i}-y_{i}\right)^{2}}
\end{aligned}
$$

Euclidean Distance Formula:

\section{Example:}

// calculate distance between lefteye center and right eye center double distanceBetweenEyes = math.sqrt(((facialFeatures[1].y facialFeatures[0].y)^2) + ((facialFeatures[1].x facialFeatures[0].x $)^{\wedge} 2$ ));

\section{RESULTS \& DISCUSSION}

Prior to code generation, the operations to be performed by the algorithm on the face image are binarization, thinning, lighting and edge detection. The Algorithm is very useful for large Databases and Data warehouses. The Algorithm will reduce the Time Complexity and Space Complexity. The RDMS Oracle is most suitable for testing and searching.

\section{Procedure for Facial Images:}

- Image is uploaded imported from database.

- The user may also prompt or upload image.

- Apply EBGM (Elastic Bunch Graph Matching) and 3-D algorithm. 
- EBGM will be applied in conjunction with a 3-D matching algorithm, in which faces are represented as graphs.

- Conversion into numeric code.

- Another algorithm is used to convert resultant image into a 14 digit code. This code will then be used for searching.

- In external requirement, Camera will be interfaced with computer system.

\section{Graphical User Interface:}

The Graphic User Interface contains personal information about the person whose image is being processed, such as First Name, Middle Names, Last name, gender, occupation, Date of Birth and the Image itself. There is also provision for the 14 Digit code returned by the Algorithm. In order to facilitate the design, we define the following terms:

Definition of thinning: The thinning operation is useful for eliminating foreground pixels from the imported binary image. It is suited for skeletonization.
Definition of Skeletonization: It is shape obtained and derived by morphological operators in image processing.

Definition of Binarization: The binarization is conversion of an image into binary image, where, a pixel is allocated either black or white; the image then has foreground color and remaining portion is allocated background color.

\section{PSEUDO CODE FOR THE DATA FLOW}

STEP 1: Scan from camera or import image from the database

STEP 2: Apply pre-process for matching code

STEP 3: Generate image code send it to GUI

STEP 4: store the code in a specified column of

\subsection{Major Points for Design}

The major points in design are scan image or import-image, enhancement and resizing of image and then thinning is applied. The 
procedure like indexing the database is followed by matching. The enhanced image is obtained for feature detection. After matching procedure, the image code is prepared and sent to GUI as well as database. With courtesy, the following design is modified form of [12] originally prepared for scanning and processing images such as fingerprints etc. as shown in figure 8 .

\section{CONCLUSION}

The Algorithm will reduce the Time Complexity and Space Complexity. The RDMS Oracle is most suitable

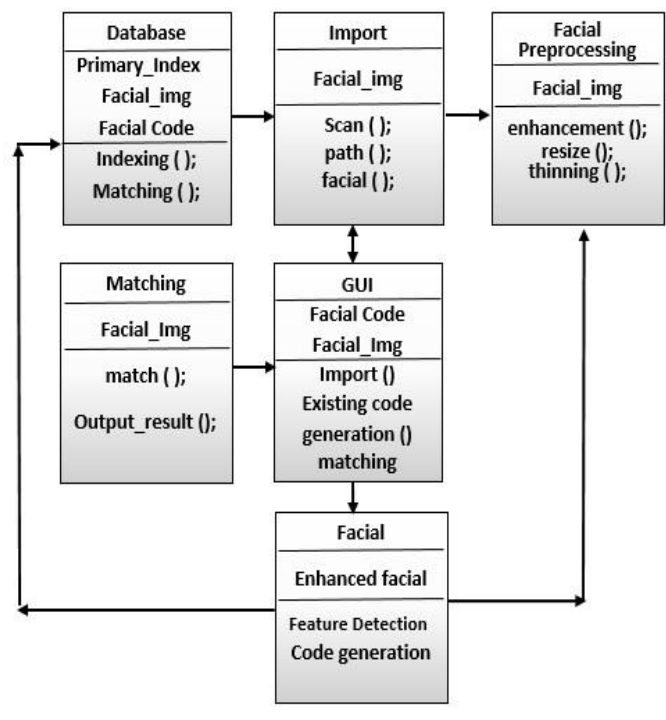

Figure 7: Data Flow Diagram for Testing and searching. The Scheme can be applied to 3D face recognition and for regenerating damaged images.

\section{Acknowledgement:}

I express my gratitude for encouragements by the Vice Chancellor and Dean (IT), Lahore Garrison University, Lahore. 


\section{REFERENCES}

[1] Aftab Ahmad Malik: "Algorithm for Coding Person's Names in large Databases/ Data Warehouses to Enhance Processing Speed, Efficiency and to Reduce Storage Requirements"; LGU Research Journal of Computer Science and Information Technology, Vol 1 issue 1, January-March 2017; ISSN 2519-7991

[2] Piyush Patel "Code to generate unique ID for face recognition using OpenCV" [closed] by Stack Overflow Community; which is a community of 6.6 million programmers; internet: www.stackoverflow.com/questi ons/16980195/code-togenerate-unique-id-for-facerecognition-using-opencv, Jun. 07, 2013 [March. 02, 2017]

[3] Robert Laganière, OpenCV 2 Computer Vision Application Programming book, Packt Publishing, 2014

[4] Shahwar H and Adnan Khan: "Face and Face Parts detection in image processing"; $L G U$ Journal of Computer Science and Information Technology,
LGURJCSIT, Vol 1, Issue 1, January-March 2017; ISSN 2519-7991

[5] Aftab Ahmad Malik: "Software for Finger Prints Storage and Retrieval of Criminal Identification System for Police", Research Journal, University of Engineering Technology, Lahore, Volume 12; No. 4; PP: 1-18

[6] Aftab Ahmad Malik: Software for Storage and Retrieval of Criminal Information for Police", Research Journal, University of Engineering Technology, Lahore, Volume 13; No. 1 PP: 1-28

[7] Brandon McKinney." Face Patterns", internet: www.pinterest.com/pin/ 512425263825056246/ [March, 2017]

[8] Laurenz Wiskott, Rolf P. Würtz and Günter Westphal. "Elastic Bunch Graph Matching", Internet:

http://www.scholarpedia.org/a rticle/Elastic_Bunch_Graph_ Matching [Feb, 2017]

[9] "Gabor Filters". Internet: http://homepages.inf.ed.ac.uk/rb f/CVonline/LOCAL_COPIES/T 
RAPP1/filter.html. 17.Nov.1998,

[March, 2017]

[10] Laurenz Wiskott, Jean-Marc

Fellous, "Face Recognition by

Elastic Bunch Graph Matching", in Intelligent Biometric

Techniques in Fingerprint and Face Recognition, CRC Press, ISBN 0-8493-2055-0, Chapter 11, pp. 355-396, (1999).

[11] M. Hess and G. Martinez, "Facial feature extraction based on the smallest univalue segment assimilating nucleus (SUSAN) algorithm," in Proceedings of the Picture Coding Symposium (PCS '04), San Francisco, Calif, USA, December 2004

[12] Mujtaba Asad, "Enhanced Security of Digital Images using Discrete Cosine \& Discrete Wavelet Transform", MS (EE) Final Thesis, GC University Lahore, 2014.

[13] J. Zafar, and Mujtaba Asad, “ Enhanced Security and Reinstatement Computing of Images Using Fragile Invisible Integer Wavelet Watermarking Scheme", in Proceedings of the World Congress on Engineering 2015 Vol I, WCE 2015, July pp, 1 - 3, London, U.K, 2015 